INSTITUT NATIONAL DE RECHERCHE EN INFORMATIQUE ET EN AUTOMATIQUE

\title{
Reducing Kernel Development Complexity In Distributed Environments
}

Adrien Lebre — Renaud Lottiaux — Erich Focht — Christine Morin

\section{$\mathrm{N}^{\circ} 6405$}

Janvier 2008

Thème NUM

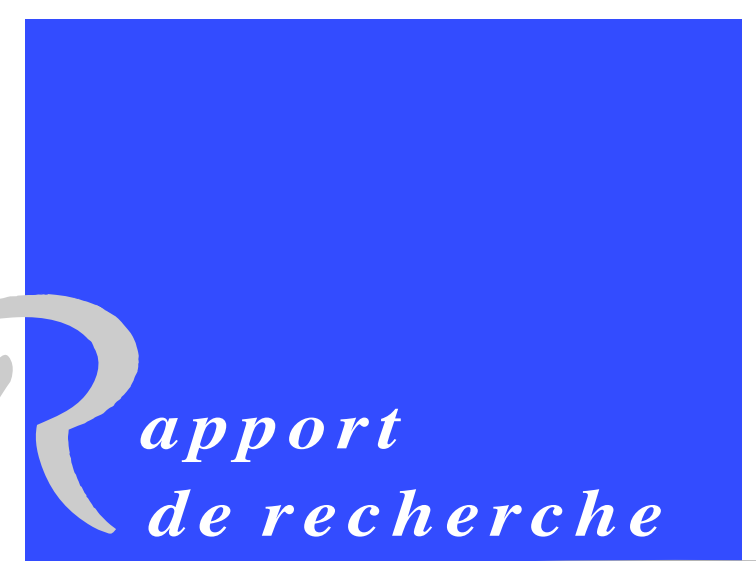





\title{
RINRIA
}

\section{Reducing Kernel Development Complexity In Distributed Environments *}

\author{
Adrien Lebre ${ }^{\dagger}$, Renaud Lottiaux ${ }^{\ddagger}$, Erich Focht $^{\S}$, Christine Morin $^{\dagger}$ \\ Thème NUM — Systèmes numériques \\ Projet PARIS
}

Rapport de recherche $n^{\circ} 6405$ - Janvier 2008 - 17 pages

\begin{abstract}
Setting up generic and fully transparent distributed services for clusters implies complex and tedious kernel developments. More flexible approaches such as user-space libraries are usually preferred with the drawback of requiring application recompilation. A second approach consists in using specific kernel modules (such as FUSE in Gnu/Linux system) to transfer kernel complexity into user space.

In this paper, we present a new way to design and implement kernel distributed services for clusters by using a cluster wide consistent data management service. This system, entitled kDDM for "kernel Distributed Data Management", offers flexible kernel mechanisms to transparently manage remote accesses, cache and coherency. We show how KDDM simplifies distributed kernel developments by presenting the design and the implementation of a service as complex as a fully symmetric distributed file system.

The innovative approach of kDDM has the potential to boost the development of distributed kernel services because it relieves the developers of the burden of dealing with distributed protocols and explicit data transfers. Instead, it allows focusing on the implementation of services in a manner very similar to that of parallel programming on SMP systems.

More generally, the use of kDDM could be exploited in almost all local kernel services to extend them to cluster scale. Cluster wide IPC, distributed namespaces (such as /proc) or process migration are some potential examples.
\end{abstract}

Key-words: Operating systems, kernel development, cluster, symmetric file system

\footnotetext{
* The authors from INRIA and NEC carry out this research work in the framework of the XtreemOS project partially funded by the European Commission under contract \#FP6-033576.

$\dagger$ INRIA Rennes Bretagne Atlantique, Rennes France - firstname.lastname@ irisa.fr

‡ KERLABS, Gevezé France - renaud.lottiaux@ kerlabs.com

$\S$ NEC, Stuttgart, Germany, efocht@ hpce.nec.com
} 


\section{Reduire la compléxité des développements noyaux dans les environnements distribués}

Résumé : La mise en œuvre de services distribués transparents et génériques dans une grappe implique des développements noyaux complexes et souvent fastidieux à finaliser. L'élaboration de bibliothèques situées en espace utilisateur est généralement préférée avec comme principal inconvénient l'obligation de recompiler les applications. Une seconde approche consiste à exploiter des mécanismes noyaux spécifiques permettant de remonter la compléxité du noyau au niveau utilisateur (le module FUSE en est un exemple). Toutefois ce type de solution peut avoir un impact négatif sur les performances.

Dans ce papier, nous présentons une nouvelle manière de concevoir et de mettre en œuvre des services noyaux distribués grâce à l'utilisation d'un service de partage cohérent de données à l'échelle des grappes. Ce système, intitulé kDDM pour "kernel Distributed Data Management", propose des mécanismes permettant de partager efficacement et de manire transparente des données noyaux entre plusieurs noeuds (le maintien en cohérence étant assuré directement par le service). Nous montrons comme l'utilisation du service kDDM permet de simplifier l'élaboration d'un service distribué en mode noyau en présentant l'implantation d'un service complexe comme celui d'un système de fichiers symétrique.

L'approche proposée dans ce document pourrait permettre d'accrotre le developpement de services distribués au niveau noyau en réduisant la charge de travail liée à la mise en place de protocol distribué ou encore celle liée à la gestion des transferts explicites des données. Les phases de développement peuvent alors être dans leur totalité consacrées à la mise en œuvre du service de manière similaire à un développement sur des systèmes SMPs.

Plus généralement, l'utilisation des kDDMs peut être exploitée dans la plupart des services noyaux usuels afin de les étendre à l'échelle des grappes. A titre d'exemple, il serait tout à fait envisageable d'étendre les mécanismes IPC, de mettre en place des espaces de nommage distribués ou encore de faciliter la migration de processus en temps réel.

Mots-clés : système d'exploitation, développement noyau, grappe, système de fichiers symétrique 


\section{Introduction}

Clusters are today a standard computation platform for both research and production. A lot of work has already been done to simplify efficient use of such architectures: batch schedulers, distributed file systems, new programming models, ... and it is likely to continue as cluster constraints are still changing: more cores per CPU socket, faster interconnects, larger scale.

Setting up generic and fully transparent distributed services for clusters implies complex and tedious kernel developments. More flexible approaches such as userspace libraries are usually preferred with the drawback of requiring application recompilations. However a lot of applications are mainly based on standards such as POSIX and recompliation is sometimes not possible (in particular for legacy codes). In such cases, distributed services have to be perfectly transparent, requiring kernel extensions. However, only few kernel mechanisms have been suggested. Current approaches consist in completing major kernel components by modules to bring back kernel complexity to user space. As an example, the FUSE module from the Gnu/Linux system makes distributed file system implementation easier. If such an approach solves the transparency issue, it impacts performance by the multiple copies from user to kernel and symmetrically. On the other side, userland cluster services are designed by levering generic libraries such as MPI making their design and their implementation much easier.

In contrast with userland, only few work has focused on providing generic layers to faciliate distributed kernel services. From our best knowledge, developers are only aware about the remote procedure call protocol. The SUN RPC model [11] is based on a client server where a node (the client) asks for a service delivered by another node (the server). This model offers some flexibility but has several drawbacks. For instance, it only enables point to point communication and is not well designed to share data structures at a fine grain.

In this paper, we present a new way to design and implement kernel distributed services for Gnu/Linux clusters by using a cluster wide consistent data management service. From our point of view, providing such a service is really innovative. First, this system, entitled kDDM for kernel Distributed Data Manager, is built with the purpose to ease the design and the development of more complex distributed services. Second, it provides a real different way to exchange and share kernel data between distinct nodes within a cluster. By using the $\mathrm{kDDM}$ mechanism, programmers are able to focus on the real purpose of cluster kernel sevices instead of dealing with distributed protocols. The kDDM infrastructure helps reducing cluster kernel development complexity to a level comparable to the development on a SMP node.

We show how kDDM makes distributed kernel developments easier by presenting the design and the implementation of a service as complex as a fully symmetric distributed file system. This file system, named kDFS enables to:

- Aggregate storage resources available within a cluster,

- Provide a unique cluster wide name-space,

- Provide cooperative cache for both data and meta-data. 


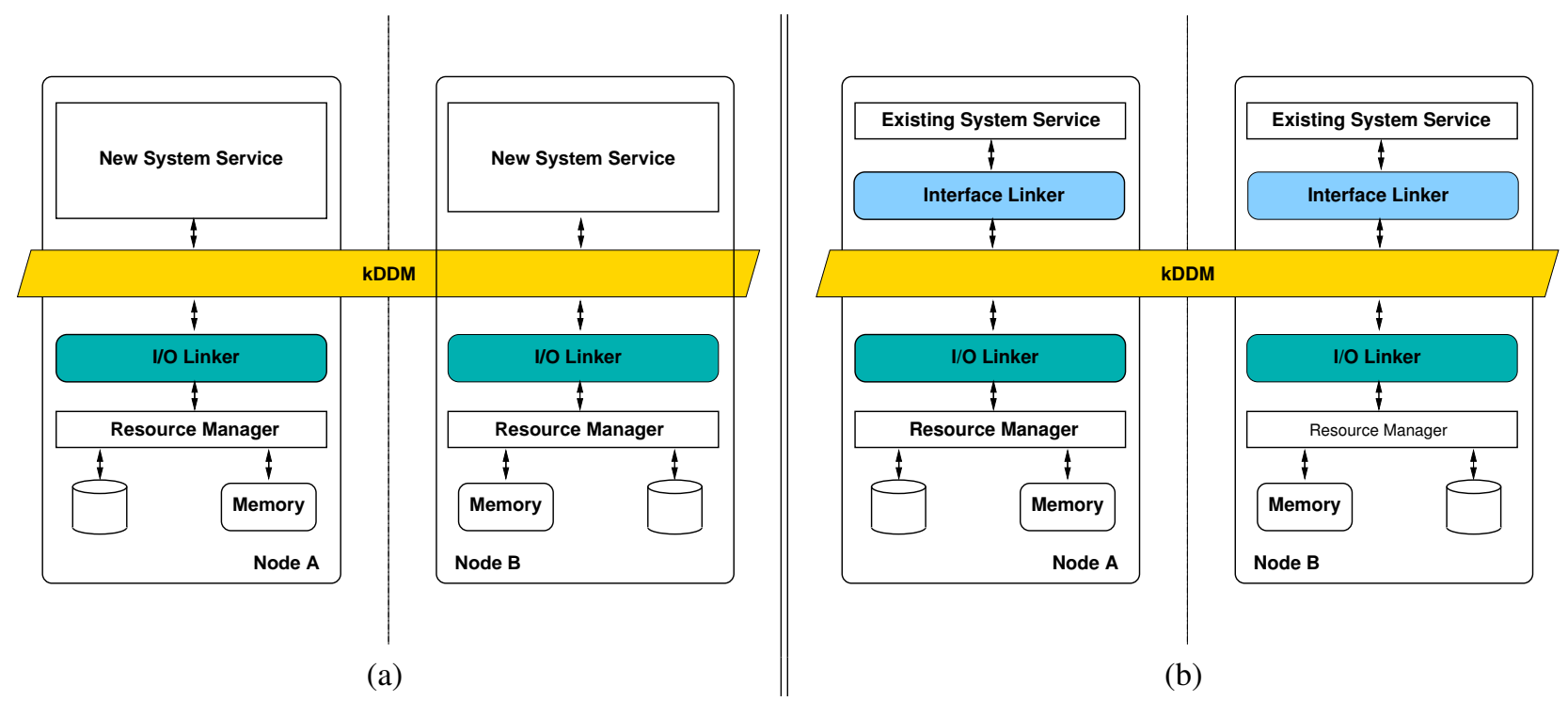

Figure 1: kDDM overview

More generally, the use of kDDM could be exploited in almost all local kernel services to extend them to cluster scale. Cluster wide IPC, distributed namespaces (such as /proc) or process migration are only a few of the candidates.

The document is organized as follows. Section 2 outlines the kDDM mechanisms. Section 3 is focused on kDFS design and its implementation. Related work is addressed in Section 4. Finally, Section 5 concludes the document and gives some perspectives.

\section{Kernel Distributed Data Manager}

The Kernel Distributed Data Manager, kDDM [8], allows consistent and transparent data sharing cluster wide. This concept was formerly called container and has been renamed to kDDM to avoid confusion with current kernel container mechanisms.

The kDDM service allows to share at kernel level collections of objects between the nodes of a cluster. In kDDM, an object is a set of bytes defined by the kDDM user (data structure, memory page content, etc). Objects of the same kind are stored in a set. An object is then identified using a pair (set identifier; object identifier).

The main goal of the kDDM mechanism is to implement distributed kernel services. Assuming that an OS could be roughly divided into two parts: (1) system services and (2) device managers, developpers could design and implement their own cluster wide services (cf. Figure 1 (a)) or extend the existing ones by using interface linkers (cf. Figure 1 (b)).

In both cases, $\mathrm{kDDM}$ sets are plugged to device managers thanks to IO linkers. 


\section{1 kDDM Sets}

A kDDM set is a collection of similar objects a kernel developer wants to share cluster wide. Each set can store up to $2^{32}$ objects. Objects are managed by the kDDM service without any assumptions on contents and semantics. In other words, developers have the opportunity to share any kind of objects.

For each kind of object to share, a new KDDM set family is created to host this kind of object. It is then possible to create several kDDM sets of the same family.

For instance, it is possible to create a kDDM set to share all the inodes of a file system. In this case we define a kDDM set family to host inodes and a new set of this family is created for each file system. In the same way, we can create a KDDM set family to host pages of system V memory segments. From this kDDM set family, we create a new set for each new system $\mathrm{V}$ segment.

Defining a new kDDM set family mainly consists in creating a new IO linker designed to manage the kind of object they are intended to host.

\subsection{IO Linkers}

Each KDDM set is associated to an IO linker depending on the family set it belongs to. For each family there is a different IO linker. During the creation of a new kDDM set, an IO linker is associated to it. Doing this instantiation, the set can be attached to a physical device or simply attached to a dedicated memory allocator (memory pages allocator, specific slab cache allocator, etc). Indeed, the IO linker defines how objects are allocated, freed, transfered from one node to another, etc.

Right after the creation of a kDDM set, the set is completely empty, i.e. it does not contain any data. Memory is allocated on demand during the first access to an object through the IO linker. Similarly, data can be removed from a kDDM set when it is destroyed, when an object is no more in use or in order to decrease the memory pressure when the physical memory of the cluster is saturated. Again, these operations are performed through the IO linker.

Examples of IO linker usage are given in Section 3, where we describe the design and the implementation of a distributed file system on top of the kDDM mechanism.

\subsection{Interface Linkers}

Existing High level kernel services can be extended to a cluster scale thanks to interface linkers. An interface linker is the glue between existing services and kDDM sets.

Moreover, it is possible to connect several system services to the same KDDM set by using different interace linkers. For instance, a kDDM set can be used to map pages of a file in the address space of a process P1 on a node A using an interface linker L1, while a process $\mathrm{P} 2$ on a node $\mathrm{B}$ can access the same $\mathrm{kDDM}$ set through a read/write interface using another interface linker L2. 


\subsection{Manipulation Functions}

Objects are handled using manipulation functions which ensure data replication and coherence. These functions can be compared to read/write locking functions. They are mainly used to create $k D D M$ critical section enabling to safely access KDDM objects regardless of data location in the cluster.

Objects stored in a kDDM set are handled using a dedicated interface. This interface is quite simple and mainly relies on three functions used to create $k D D M$ critical sections: get, grab and put.

The get function is close to a read-lock: it places a copy of the requested object in local memory and locks it cluster wide. This locking ensures that the object can not be written on any other node in the cluster. However, concurrent read accesses are allowed.

The grab function is close to a write-lock: it places a copy of the requested object in local memory and locks it cluster wide. No other concurrent access (read or write) is allowed cluster wide (cf. Section 2.5).

The put function is used to unlock an object.

In addition to these main functions, a few other ones are used, such as remove, flush or sync. The remove function removes an object from the memory cluster wide since the flush function only removes an object from local memory and ensures that there is at least one node still hosting a copy.

Finally, the sync function synchronizes an object with its attached physical device, if any. This function is useful in the context of a file system, to write back data to disk.

\subsection{Replication And Coherence}

During a $\mathrm{kDDM}$ critical section, object data is stored in the memory of the node doing a get or a grab and can be accessed using regular memory operations. Outside a kDDM critical section, there is no guarantee that the object is still present in node local memory. In most cases the data is still present, but the kDDM semantics does not allow to access this data outside a kDDM critical section.

As suggested in the previous section, objects are moved from one node to another during a grab operation and can be replicated on different nodes for efficiency reasons during a get. Replication introduces a data coherence issues. Coherency is managed using an invalidation on write protocol, derived from the one presented by Kai Li [5]. The grab function, semantically equivalent to a write, is used to catch object modifications. In this case, all the existing remote copies are invalidated before the grab returns. During the grab critical section, the object is locked on the node (where the grab has been done) and cannot be moved or replicated.

\section{Kernel Distributed File System}

In order to show the interest of generic distributed kernel mechanisms such as the kDDMs, we chose to design and implement a service as complex as a fully symmetric file system. To our knowledge, only few distributed file systems have been designed with 
fully symmetric constraints $[1,6]$. The implementation complexity of such systems is generally dissuasive and the current trend consists in designing and implementing distributed file systems composed by one or two meta-data servers and several I/O servers $[3,7,10]$. By dividing meta-data and data management, such solutions, entitled parallel file systems, make the implementation of the client stack easier. However such a model can lead to a non balanced architecture between distinct nodes. For example: the scalability limitation imposed by a single metadata server does not exist in a fully symmetric file system.

Thanks to kDDM, we were able to quickly design and implement a fully symmetric file system providing a cooperative cache for both data and meta-data. To our knowledge, providing such a kernel distributed file system is innovative since this new proposal mainly focuses on real file system issues instead of dealing with distributed protocols.

The design of kDFS relies on two main concepts:

- Using native file systems available on each node (avoiding block device management),

- Using kDDM sets to provide a unique and consistent cluster wide name space.

The use of kDDM sets facilitates the implementation of a global distributed cache with Posix semantics since kDDM mechanisms directly ensure meta-data and data consistency.

Figure 2 illustrates the overall architecture of kDFS.

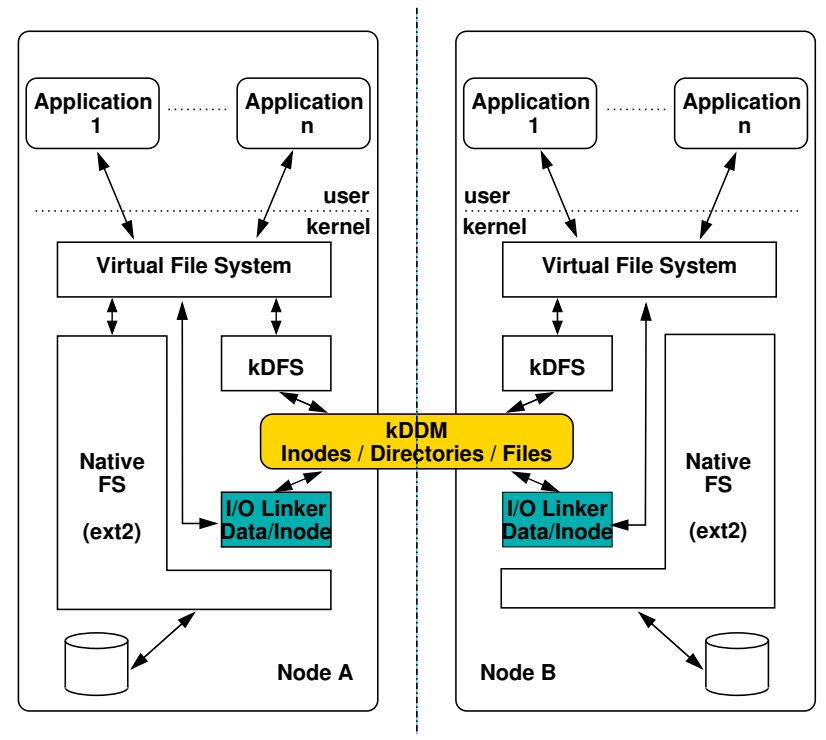

Figure 2: Overview of the kDFS system

After describing how kDFS uses native file systems to store data, we present how kDDM mechanisms have helped kDFS be implemented with elegance and simplicity. 


\subsection{Disk Layout}

To avoid block device dependencies and make storage management easier, we have chosen to build kDFS upon native file systems provided by the cluster nodes. As a consequence, each node has to specificy if it takes part in the kDFS storage space or not. Storage space provided by a particular cluster node is considered as a kDFS partition only if it has been kDFS formatted. As kDFS does not directly manipulate block devices, a kDFS partition actually refers to a local directory with a particular hierarchy. This section introduces different sub-directories and different files contained in a $\mathrm{kDFS}$ partition.

To format a directory which can be used afterwards in the kDFS storage space, administrators have to use the mkfs. kdfs command. This command takes two arguments: DIR_PATHNAME and ROOT_NODEID. The first one corresponds to the absolute path to store kDFS meta-data and data, the second one is the node identifier for the kDFS root entry (the node that stores the kDFS root inode).

mkfs.kdfs creates the kDFS "superblock" file (...) for the node. This file is stored on the local native file system in the given directory. If the current node identifier (last byte of the IP address) equals to the given $i d, \operatorname{mkfs} . \mathrm{kdfs}$ creates the root entry.

Table 1 describes the creation of a kDFS structure distributed between two nodes:

\begin{tabular}{|l|l|}
\hline On node A: (nodeid = 1) & on Node B: (nodeid = 2) \\
\hline mkfs.kdfs /PATH1 1 & mkfs.kdfs /PATH2 1 \\
\hline $\begin{array}{l}\text { Create kDFS local '...' } \\
\text { Create kDFS root entry }\end{array}$ & Create kDFS local '...' \\
\hline
\end{tabular}

Table 1: kDFS structure creation (two nodes)

For each entry (a directory or a file) of the kDFS hierarchy, a "native" directory is created on one kDFS partition. This directory contains two files:

- The . met a file that stores meta-data associated with the entry (size, timestamp, rights, striping information,...)

- The. content that stores real data (directory an file contents).

The name of the "native" directory is defined by the kDFS local inode identifier (each kDFS superblock contains an identifier bitmap to define the next free inode $i d$ ).

To avoid scalability issues with large directories, we have arbitrarily chosen to sort each kDFS partition in groups of one hundred entries. For instance, when mk $\mathrm{s}$. kdf $\mathrm{s}$ creates the kDFS root entry (the first entry of the kDFS hierarchy), the command first creates a sub-directory 'DIR_PATHNAME/0-99/'. Then, it creates the corresponding "native" directory which is the DIR_PATHNAME / 0-99/1/ directory. Finally, the file '. meta' and the file'. content' are created inside this latest directory.

Every hundred entries, kDFS adds a new sub-directory corresponding to the appropriate range ('DIR_PATHNAME/100-199/', 'DIR_PATHNAME/200-299/,,...). 
Once the partition is formatted, users can access the kDFS file system thanks to the conventional mount command:

mount -t kdfs ALLOCATED_DIR/NONE MOUNT_POINT

where ALLOCATED_DIR corresponds to the native file system directory formatted with mkfs.kdfs and MOUNT_POINT is the traditional mount point.

Table 2 describes kDFS mounting procedure from two nodes:

\begin{tabular}{|l|l|}
\hline On node A: $($ nodeid = 1) & on Node B: $($ nodeid = 2) \\
\hline mount /PATH1 /mnt/kdfs & mount /PATH2 /mnt/kdfs \\
\hline
\end{tabular}

Table 2: Mount kDFS partitions

/mnt is now a global kDFS namespace for both nodes

Since files and directories are stored in a distributed way on the whole cluster, we need mechanisms to find the kDFS entry point and thus be able to join the file system. kDFS provides two ways of retrieving the root inode. The first one is based on the superblock file stored in the formatted kDFS partition. As mentioned, the kDFS superblock file provides several information including the kDFS root inode id. Thus, when a mount operation is done, the '...' file is read from 'ALLOCATED_DIR' and the root inode $i d$ is used to retrieve the kDFS root entry. The second mechanism relates to diskless nodes or nodes which do not want to take part in the kDFS physical structure. In such a case, users do not specify a "device" but have to provide the kDFS root inode id as an additional mount parameter.

Moreover, we plan to take advantage of the kDFS superblock file to add some QoS parameters (such as the allowed storage space, rights) for each kDFS "partition".

\subsection{File System Architecture}

The use of $\mathrm{kDDM}$ in our symmetric file system enables to propose a simple design based on two layers. The highest one, in charge of forwarding local requests to the $\mathrm{kDDM}$ serivce, is directly plugged under the VFS. The lowest one, composed by the different I/O linkers, applies kDDM requests on proper kDFS partitions

The first version of kDFS has been implemented using three families of kDDM sets:

- Inode $k D D M$ set, one cluster wide. It provides a cache of inodes recently accessed by processes.

- Dir kDDM set, one per directory. Each Dir kDDM set contains directory entries (roughly names of subdirectories and files).

- File $k D D M$ set, one per file. It stores data related to the file contents.

Figure 3 depicts the kDDM entities for several kDFS entries. To make reading and understanding easier, we present in Table 3 a potential representation of the regular files for each kDFS entries mentioned in Figure 3. 


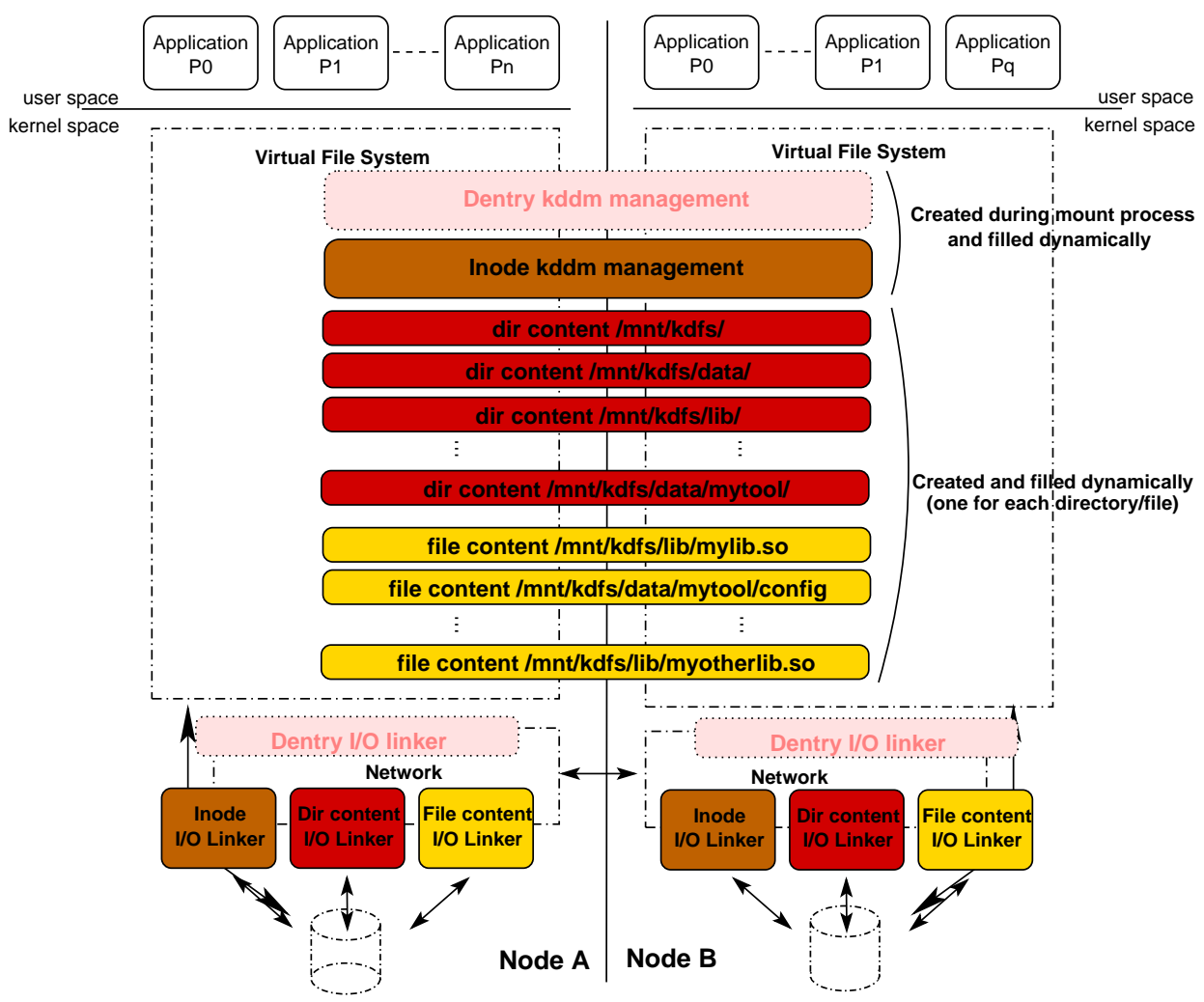

Figure 3: KDFS internal layers

\begin{tabular}{|c|c|c|c|}
\hline \multicolumn{2}{|c|}{$\begin{array}{l}\text { On node A: }(\text { nodeid }=1) \\
\text { DIR_PATHNAME: /PATH1 }\end{array}$} & \multicolumn{2}{|c|}{$\begin{array}{l}\text { on Node B: }(\text { nodeid }=2) \\
\text { DIR_PATHNAME: /PATH2 }\end{array}$} \\
\hline mount $/ \mathrm{P}$ & $1 / \mathrm{mnt} / \mathrm{kdfs}$ & mount /PATH2 & $/ \mathrm{mnt} / \mathrm{kdfs}$ \\
\hline$" / \mathrm{mnt} / \mathrm{kdfs} / "$ & $\begin{array}{l}\text { /PATH1/0-99/1/.meta } \\
\text { /PATH1/0-99/1/.content }\end{array}$ & $" / m n t / k d f s / d a t a / "$ & $\begin{array}{l}\text { /PATH2/0-99/1/.meta } \\
\text { /PATH2/0-99/1/.content }\end{array}$ \\
\hline "/mnt/kdfs/lib" & $\begin{array}{l}\text { /PATH1/0-99/2/.meta } \\
\text { /PATH1/0-99/2/. content }\end{array}$ & "/mnt/kdfs/data/mytool/" & $\begin{array}{l}\text { /PATH2/100-199/104/.meta } \\
\text { /PATH2/100-199/104/.content }\end{array}$ \\
\hline "/mnt/kdfs/lib/mylib.so" & $\begin{array}{l}\text { /PATH1/200-299/280/.meta } \\
\text { /PATH1/200-299/280/.content }\end{array}$ & "/mnt/kdfs/data/mytool/config" & $\begin{array}{l}\text { /PATH2/100-199/105/.meta } \\
\text { /PATH2/100-199/105/.content }\end{array}$ \\
\hline & $\begin{array}{l}\cdots \\
\ldots\end{array}$ & "/mnt/kdfs/lib/myotherlib.so" & $\begin{array}{l}\text { /PATH2/100-199/180/.meta } \\
\text { /PATH2/100-199/180/.content }\end{array}$ \\
\hline
\end{tabular}

Table 3: Translation between kDFS entries and regular files on hard drives The global kDFS namespace is distributed on two nodes. 
Next sections introduce each of these three families of kDDM sets. A fourth kDDM set is depicted in Figure 3: the dentry kDDM set. This unique kDDM set provides a distributed cache to manage all dentry objects. It is currently not implemented and requires deeper investigation.

\section{4 kDFS Inode Management}

kDFS inode management relies on one global kDDM set. This set exploits a dedicated I/O linker for inserting/removing and maintaining each kDFS entry on the corresponding local file system (sub-directory creation/deletion and updating of the '. met a file'). The inode set is created during the first kDFS mount operation within the cluster. At the beginning, it only contains the kDFS root inode. Then, when a process wants to access one file/directory, its corresponding kDFS inode is added into the set (providing by this way a fully distributed inode cache). All file/directory creations are performed locally whenever possible. That means, when a process wants to create a new file or a directory, $\mathrm{kDFS}$ looks for a kDFS partition. If there is one directly available on the node, a new inode identifier is obtained from the superblock file. Otherwise, kDFS stores the new entry on the same node as the parent directory.

When a mount operation is done, the root inode identifier is used to retrieve the root inode structure directly inside the inode $\mathrm{kDDM}$ set. If the $\mathrm{kDDM}$ set already exists, the structure is already cached and the inode is simply returned to the node. Otherwise, the request is forwared by the $\mathrm{KDDM}$ service to the proper $\mathrm{I} / \mathrm{O}$ linker within the cluster. Finally, the I/O linker exploits the inode id to retrieve required information from the hard drive.

KDFS inodes are currently based on 32 bits, the 8 MSB bits provide the node id within the cluster and the 24 LSB ones correspond to the local $i d$.

The inode management proposal has some scalability limitations due to our current implementation of a 32 bits inode. $\mathrm{kDFS}$ can federate at most 256 nodes and manage only one kDFS partition with a maximum of $2^{24}$ files for each node. We plan to fix this issue by extending the inode size to 64 bits as it is already done by several file systems (XFS, NFS, ...).

\section{5 kDFS Content Management}

Since kDFS file hierarchy is based on native file systems, both directories and files are simply stored as regular files. In contrast to traditional file systems, the management of a large kDFS directory containing a huge number of directory entries is similar to the management of a kDFS file content. Regardless of the kDFS entry, its content is stored in its respective '. content' file.

After briefly describing directory and file content manipulation, this section focuses on optimization mechanisms such as read-ahead or write-behind and how they could be exploited to improve the overall performance of kDFS. The last paragraph introduces data-striping and redundancy mechanisms. 


\section{1 kDFS Directory Management}

When an application tries to list the contents of a directory stored in the kDFS storage space, kDFS creates a new directory kDDM set. This new set is linked to the inode object stored in the inode kDDM set and caches all directory entries on a page basis. In other words, all file and subdirectory names stored in the directory are loaded in objects of this new kDDM set (one object corresponding to one page). After that, all filename manipulations such as create, rename and remove apply modifications of these pages. The associated dir I/O linker is in charge of propagating changes to the proper hard drive (into the '. content' file)

\section{2 kDFS File Management}

In this first prototype, $\mathrm{kDFS}$ file management is similar to directory management: when an application tries to access a file $f$, if $f$ is not already "cached", kDFS creates a new file kDDM set. As for directory management, this new set is linked to the corresponding inode object of the file $f$. The associated file I/O linker is in charge to read/write data from/to the . content file of $f$ and remove/put pages in the set.

\subsection{Read-ahead And Write Behind}

Caching is a widespread technique used to reduce the number of accesses to hard drives and improve performance of the I/O system [2]. In a local context, such mechanisms mainly appear at high level in the I/O stack (in the VFS for UNIX like OS, cf. Figure 4). Due to kDFS placement within the I/O stack and the KDDM design, cache mechanisms could be applied twice in KDFS: at low level (when I/O linkers access the native file system, cf. 1 in Figure 4) and at high level (when linkers access kDFS, cf. 2 in Figure 4). At the first sight, it seems natural to exploit such techniques to improve performance at any level they can be applied. However, after a deeper study, the naive use of readahead and write-behind at high level lead to a negative impact on both efficiency and consistency.

At low level, read-ahead and write-behind strategies only depend on the local file system and can be compared to internal buffer caches available in hard drives. In other words, if a process reads or writes in one kDFS file stored on a remote node, cache mechanisms applied to the remote I/O linker do not change the consistency within the associated $\mathrm{kDDM}$ set.

At high level, it is a bit more sophisticated. Interest and impact of each strategy require to be tackled one by one.

- The read-ahead strategy has been suggested to mask disk latencies. This technique is mainly based on the sequential behaviour of readers and can even decrease performance in case of random accesses (wrong pages are prefetched). In a distributed context such as the high layers of kDFS, the use of read-ahead can either improve performance in some cases or impact network traffic in some other ones. Typically, for a distributed application, some useless chunks of one file may be prefetched from several nodes. These pages go through the network 


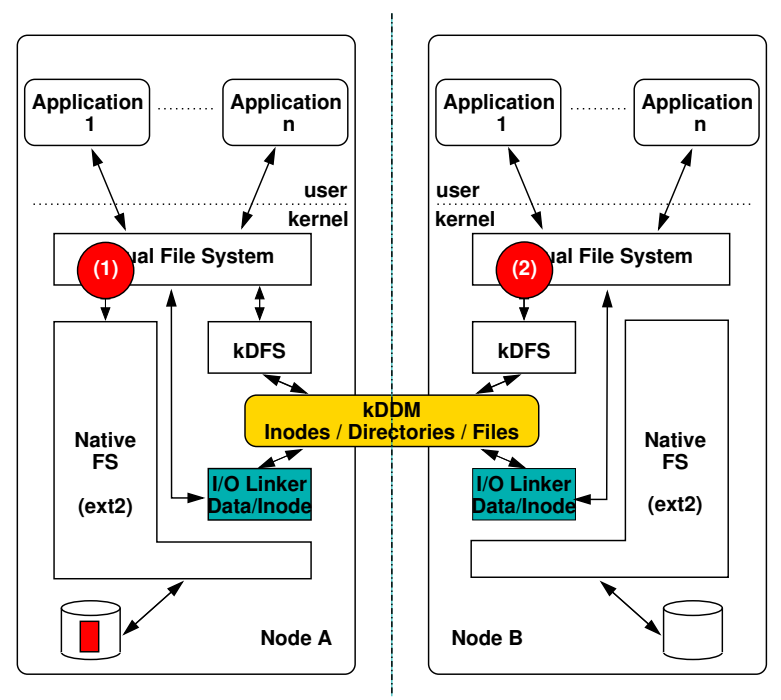

Figure 4: Cache in kDFS

several times, increasing the network congestion probability. Thus, a performance tradeoff between prefetch mechanisms and network congestion should be defined. The disk latency is about $9 \mathrm{~ms}$ in current harddrives whereas network latency tends to be negligible at cluster level $(i 1 \mu s)$. So, we have chosen to leave aside read-ahead at high level for the moment.

- The write-behind strategy at high level is a real issue. From the performance point of view, such a technique is crucial to avoid page migration from one node (high level) to a remote one (low level). Let's consider an process that is writing to a remote file byte per byte. A write-through strategy generates a page migration to acknowledge each write operation whereas a write-behind strategy significantly reduces network traffic by aggregating several writes before flushing data to low level. However, in the event of a failure, such an approach may lead to an inconsistency. Indeed if a crash occurs on a node writing into the file, data contained in the write-behind buffer is simply lost, although these writes have been acknowledged. To avoid such critical situations, we have decided to only implement a write through strategy in the first version of kDFS: each write is directly propagated to the corresponding I/O linker. We currently work on the design of derived mechanisms to improve kDFS performance in case of write requests.

\subsection{Striping And Redundancy}

The objectives of striping and redundancy policies are twofold: first, to balance I/O requests over several nodes in order to decrease the amount of requests per node (and thus increase the scalability) and second to benefit from aggregating throughputs provided by several nodes. Indeed in a conventional cluster, some dedicated nodes attached to RAID devices are exploited to deliver the expected bandwidth for each application. In 
our view of a cluster file system, each node can provide its storage support which corresponds in most of cases to one traditional hard drive with a throughput peak around $60 \mathrm{MBytes} / \mathrm{sec}$. In such a case, striping and replication policies ${ }^{1}$ are mandatory.

kDFS has been designed for providing two striping modes: the first one is automatic and transparent whereas the second one is based on user parameters. In the transparent mode, all data is stored locally. That is, all write requests are propagated on local hardrives. Two cases should be considered, sequential and parallel access:

- Sequential access: only large files (out-of-core) could suffer from such an approach. A way to solve this issue is to stripe a file on multiple hard drives when its size is bigger than a defined threshold.

- Parallel access: the performance should be excellent. For instance, in MPI applications, each MPI instance writes its data locally according to the striping policy. This policy is selected by the application (CYCLIC, BLOCK/ BLOCK, ...). From the file system point of view, there is no fixed striping size. This mode should improve the performance since it avoids all striping issues which may appear when the file striping does not correspond to the pattern of the application.

In the "user" mode, each user is able to define a specific striping policy on a per file or per directory basis. Currently, we have almost finalized the design of the automatic approach and the implementation is under development. We have chosen to focus our effort on implementing the transparent mode since, in contrast to the second mode, it does not require to extend the POSIX API.

All placement information for each file is stored within the associated meta-data file (cf. Section 4). The striping geometry is based on an "object" granularity (from 1 to $n$ block). For instance, the meta-data lists all objects: a first object which is $p$ blocks long is stored on the first drive of node $x$ and a second one, $q$ blocks long, is available on the drive of node $y, \ldots(p \neq q)$. Thus, when an Inode I/O linker retrieves inode data, it also retrieves geometry parameters. kDFS can then exploit these values to notify $\mathrm{kDDM}$ service where to find proprer I/O linkers (based on the part of the directory/file content).

The use of replication is also a well-known approach to improve efficiency. Instead of accessing only one file stored on one hard drive, clients are balanced on replicas available on distinct nodes. In the particular case of kDFS such an approach is not required. Indeed, when a client accesses a file, this file is immediately stored in the associated kDDM set. Thus, all subsequent accesses are directly satisfied by the cluster wide cache and do not reach hard drives. Based on the current trend of RAM memory size increasing, we can imagine that each node will soon have a memory size close to $10 \mathrm{~GB}$. Deploying the kDFS solution on such a cluster will provide a large and efficient cluster wide buffer cache.

\footnotetext{
${ }^{1}$ We distinguish striping policies to improve performance from the ones that target fault tolerance aspects. Fault tolerant mechanisms are not addressed in this paper.
} 


\section{Related Work}

While distributed applications programmers can choose between a multitude of parallelization concepts, libraries and languages, infrastructure extensions for supporting distributed operating systems are rather rare. Most distributed services such as the Linux ones (the global filesystems Redhat GFS and Oracle's OCFS2, the distributed lock manager or the clustered logical volume manager) are each using their own protocols for communicating across nodes and have their own notion and view of what group of nodes they regard as "the cluster".

All these services are implemented on top of the socket interface, with no cluster abstraction or communication layer in between.

A component worth mentioning as piece of infrastructure specially built to ease distributed computing is SunRPC [11]. It is used heavily in the network file-system NFS implementations and offers mechanisms to register, locate and invoke remote procedures, while transfering data in the exchangeable data format XDR, allowing nodes of different architectures to interoperate transparently. Programming with RPCs has been adopted widely in both user and kernel space and has contributed significantly to the development of distributed computing.

A further step towards distributed operating systems is the introduction of cluster aware network stacks like the Transparent Inter Process Communication protocol TIPC [9]. It uses a cluster oriented addressing scheme, implements features like reliable multicast, cluster membership with subscription to membership change notifications, cluster-wide services and name-spaces. TIPC provides a socket interface to ease transition of socket based codes, as well as a raw kernel-only interface which allows full control over all features. kDDM is leveraging TIPC by using it as low level cluster communication infrastructure.

All former solutions are based on the message passing model. Another approach close to the kDDM one has been proposed by the PLURIX project [4]. Built on top of distributed JAVA objects, this system shows that a shared object model can make the design of distributed services easier. The PLURIX object consistency is based on a transactional model whereas kDDM service exploits an invalidation-on-write protocol.

\section{Conclusion And Future Work}

The paper has introduced kDDM as infrastructure component suitable for building cluster-wide services in a simple and natural way. The symmetric filesystem kDFS was presented as one highly complex distributed service leveraging kDDM functionality. Its implementation shows how the complexity of managing distributed data transfers and protocols can be reduced dramatically by using the kDDM's distributed shared objects concept.

The paper also aimed at pointing to a general method of designing and implementing distributed services by extending their well-known non-distributed variants with kDDM functionality. This methodology has been applied in the past years and lead to the Kerrighed single system image system, one of the most complete implementations 
of SSI. Kerrighed is a distributed operating system where all distributed components are implemented on top of the KDDM infrastructure.

Future work aims at providing kDDM as component loadable as module for normal unpatched Linux kernels, and increasing the number of stand-alone distributed services built on top of kDDM. Work on the symmetric file system $\mathrm{kDFS}$ will continue ${ }^{2}$, next planned steps being: improvement of stability, evaluation and tuning of performance. For instance, we plan to set up a dedicated scheduling to handle/optimize kDDM exchanges/communications as several kernel components use it.

\section{References}

[1] Thomas E. Anderson, Michael D. Dahlin, Jeanna M. Neefe, David A. Patterson, Drew S. Roselli, and Randolph Y. Wang. Serverless network file systems. Computer Science Division, University of California at Berkeley, CA 94720, 1995.

[2] Pei Cao, Edward W. Felten, Anna R. Karlin, and Kai Li. Implementation and performance of integrated application-controlled file caching, prefetching, and disk scheduling. ACM Transactions on Computer Systems, 14(4):311-343, 1996.

[3] Philip H. Carns, Walter B. Ligon III, Robert B. Ross, and Rajeev Thakur. PVFS: A parallel file system for linux clusters. In Proceedings of the 4th Annual Linux Showcase and Conference, pages 317-327, Atlanta, GA, October 2000. USENIX Association.

[4] R. Goeckelmann, M. Schoettner, S. Frenz, and P. Schulthess. Plurix, a distributed operating system extending the single system image concept. In Canadian Conference on Electrical and Computer Engineering, 2004. Vol.4, pages 1985-1988, 2004.

[5] Kai Li. Shared Virtual Memory on Loosely Coupled Multiprocessors. PhD thesis, Yale University, September 1986.

[6] Qun Li, Jie Jing, and Li Xie. Bfxm: a parallel file system model based on the mechanism of distributed shared memory. SIGOPS Operating Systems Review, 31(4):30-40, 1997.

[7] Pierre Lombard, Yves Denneulin, Olivier Valentin, and Adrien Lebre. Improving the performances of a distributed nfs implementation. In Proceeding of the 5th International Conference on Parallel Processing and Applied Mathematics, Czestochowa, Poland, September 2003.

[8] Renaud Lottiaux and Christine Morin. Containers: A sound basis for a true single system image. In Proceeding of IEEE International Symposium on Cluster Computing and the Grid (CCGrid '01), pages 66-73, Brisbane, Australia, May 2001.

\footnotetext{
${ }^{2}$ The first prototype is available at http: / / www . kerrighed.org/wiki/index.php/KernelDevelKdFS
} 
[9] John Maloy. Tipc: Providing communication for linux clusters. In In Proceedings of the Linux Symposium, July 21st-24th, Ottawa, Ontario, Canada, pages 347356, 2004.

[10] Phil Schwan. Lustre : Building a file system for 1,000-node clusters. In Proceedings of the Linux Symposium, Ottawa, July 2003.

[11] Rpc: Remote procedure call protocol specification version 2. RFC1057 Internet Request For Comments, June 1988. 
Unité de recherche INRIA Rennes

IRISA, Campus universitaire de Beaulieu - 35042 Rennes Cedex (France)

Unité de recherche INRIA Futurs : Parc Club Orsay Université - ZAC des Vignes 4, rue Jacques Monod - 91893 ORSAY Cedex (France)

Unité de recherche INRIA Lorraine : LORIA, Technopôle de Nancy-Brabois - Campus scientifique 615, rue du Jardin Botanique - BP 101 - 54602 Villers-lès-Nancy Cedex (France)

Unité de recherche INRIA Rhône-Alpes : 655, avenue de l'Europe - 38334 Montbonnot Saint-Ismier (France)

Unité de recherche INRIA Rocquencourt : Domaine de Voluceau - Rocquencourt - BP 105 - 78153 Le Chesnay Cedex (France)

Unité de recherche INRIA Sophia Antipolis : 2004, route des Lucioles - BP 93 - 06902 Sophia Antipolis Cedex (France) 